\title{
Multiple colonization with carbapenem-resistant Gram-negative bacteria acquired in India and transferred to Switzerland
}

\author{
Linda Mueller ${ }^{1,2} \cdot$ Cornelia Ottiger $^{3} \cdot$ Anthony Demord $^{1,2} \cdot$ Laurent Poirel $^{1,2,4} \cdot$ Patrice Nordmann $^{1,2,4,5}$
}

Sir,

Antimicrobial resistance is a serious and growing threat for global public health. In the European Union, Iceland and Norway, infections caused by antibiotic-resistant organisms are estimated to be responsible of 25,000 deaths per year [1].

A Swiss expert group on infectious diseases and hospital hygiene (Swissnoso) estimates that approximately 70,000 nosocomial infections occurs yearly, among which, nearly $2.9 \%$ end fatally for the patients [2]. In addition, attributable mortality to multidrug-resistant bacteria, mostly Gramnegatives, has been recently reported from Switzerland [3].

The most clinically-significant and emerging resistant determinants are: (1) the extended-spectrum $\beta$-lactamases (ESBL) that confer resistance to all $\beta$-lactams except cephamycins and carbapenems, (2) the carbapenemases that confer resistance to virtually all $\beta$-lactams, and (3) the $16 \mathrm{~S}$ rRNA methylases that confer resistance to all aminoglycosides by methylation of the aminoglycosides target, namely the $16 \mathrm{~S}$ ribosomal RNA.

We report here the colonization/infection of a patient hospitalized in India with six unrelated multidrug-resistant Gram-negative isolates who was transferred to Switzerland.

Laurent Poirel

laurent.poirel@unifr.ch

1 Emerging Antibiotic Resistance Unit, Medical and Molecular Microbiology, Faculty of Science and Medicine, University of Fribourg, Chemin du Musée 18, 1700 Fribourg, Switzerland

2 Swiss National Reference Center for Emerging Antibiotic Resistance (NARA), University of Fribourg, Fribourg, Switzerland

3 Institute for Laboratory Medicine, Cantonal Hospital of Aarau, Aarau, Switzerland

4 INSERM European Unit (IAME, France), University of Fribourg, Fribourg, Switzerland

5 University Hospital Center and University of Lausanne, Lausanne, Switzerland
In November 2018, a 47-year-old man was repatriated from India to the tertiary care cantonal hospital of Aarau, Switzerland, after 2 weeks of hospitalization in three Indian hospitals (Goa region) for venous thrombosis, followed by severe nephropathy, likely related to rhabdomyolyses due to immobilization. Upon admission in Switzerland, he presented an acute kidney failure and was admitted for haemodialysis. He had multiple abscesses (lateral thoraces, abdomen, gluteal and rectal), likely caused by traumatic immobilisation and/or septic thrombosis, which were debrided at the Aarau hospital.

As any patient having been hospitalized abroad, he was screened at the entrance for methicillin-resistant Staphylococcus aureus (MRSA), vancomycin-resistant Enterococci (VRE), extended-spectrum $\beta$-lactamase (ESBL)-producing and carbapenem-resistant Gram-negative bacteria. Urine, faeces and a pooled swab from nose, throat, axilla and groin were withdrawn and sent for screening of ESBL production on CHROMagar Orientation (CHROMagar TM), for carbapenem resistance on CHROMagar mSuperCARBA, for MRSA on CHROMagar MRSA and for VRE on CHROMagar VRE plates. No growth was observed on MRSA and VRE plates. However, different colony types grew on the ESBL as well as on the carbapenem-resistant screening plates. A total of six multidrug-resistant Gram-negative bacterial isolates were obtained, being either colonizing or infecting agents (Table 1). Antimicrobial susceptibility testing was performed by disk diffusion assay (Sanofidiagnostic Pasteur, France), gradient strips (bioMérieux, Liofilchem), and broth dilution techniques were used to assess minimal inhibitory concentrations (MIC). EUCAST guidelines were used to interpret antimicrobial susceptibility [4]. Pan-resistant Escherichia coli, Klebsiella pneumoniae, Enterobacter cloacae, Providencia rettgeri, Acinetobacter baumannii and Pseudomonas aeruginosa were found. All isolates but K. pneumoniae, showed resistance to ertapenem, and resistance or intermediate susceptibility to meropenem and imipenem. Additionally, all isolates showed resistance 
Table 1 Resistance determinants of the six Gramnegative bacterial isolates

\begin{tabular}{lllll}
\hline Strain & $\beta$-lactamase resistance determinant & & $\begin{array}{l}\text { Non } \\
\beta \text {-lactamase } \\
\text { resistance } \\
\text { determinant }\end{array}$ \\
\cline { 2 - 5 } & Carbapenemase & ESBL & Cephalosporinase & $\begin{array}{l}\text { 16S rRNA } \\
\text { methytrans- } \\
\text { ferase }\end{array}$ \\
\hline E. coli $(\mathrm{C}+\mathrm{I})$ & $\mathrm{NDM}-5$ & $\mathrm{CTX}-\mathrm{M}-15$ & - & - \\
K. pneumoniae $(\mathrm{C}+\mathrm{I})$ & - & $\mathrm{CTX}-\mathrm{M}-15$ & - & - \\
$\begin{array}{l}\text { P. rettgeri }(\mathrm{C}) \\
\text { E. cloacae }(\mathrm{C})\end{array}$ & $\mathrm{NDM}-5$ & - & $\mathrm{CMY}$ & ArmA \\
$\begin{array}{l}\text { P. aeruginosa }(\mathrm{C}) \\
\text { A. baumannii }(\mathrm{C}+\mathrm{I})\end{array}$ & $\mathrm{NDM}-1$ & $\mathrm{CTX}-\mathrm{M}-15$ & - & ArmA \\
\hline
\end{tabular}

$C$ patient colonized, $C+I$ patient colonized and infected (rectal abscess) to expanded-spectrum cephalosporins (ceftazidime, cefotaxime, cefepime), with the exception of the $P$. rettgeri and $P$. aeruginosa isolates that were of intermediate susceptibility and susceptible to cefepime, respectively. The Rapid Carba NP test for Enterobacterales [5] and P. aeruginosa [6] and the CarbAcineto NP test for A. baumannii [7] were performed to detect carbapenemase activities. All isolates except the $K$. pneumoniae isolate gave positive results. PCR amplification followed by sequencing identified production of carbapenemases in all those isolates, being either NDMor VIM-type metallo- $\beta$-lactamases (Table 1). All strains remained susceptible to colistin according to the results of Rapid Polymyxin NP test [8], except the P. rettgeri isolate, that bacterial species being naturally resistant to polymyxins.

In addition, three isolates (E. coli, K. pneumoniae, $E$. cloacae) exhibited a synergy between clavulanic acid and aztreonam and/or cefepime. Results of the Rapid ESBL NP test [9] together with PCR amplification and sequencing showed that these isolates produced an ESBL of CTX-M type (Table 1). Only P. rettgeri showed a restoration of the susceptibility to ceftazidime and cefotaxime in the presence of cloxacillin, suggesting the additional role of the overexpression of a cephalosporinase in the susceptibility pattern, i.e., the resistance to expanded-spectrum cephalosporins. PCR amplification revealed the presence of a plasmidencoded cephalosporinase $b l a_{\mathrm{CMY}}$ gene in that latter isolate.

Since $P$. rettgeri and E. cloacae were also resistant to amikacin, gentamicin, tobramycin and netilmicin, 16S rRNA methyltransferase genes were searched by PCR amplification. The 16S rRNA methyltransferase gene armA was identified in both isolates (Table 1). Finally, all isolates showed resistance to fluoroquinolones.

Taking into account the diversity of multidrug-resistant isolates, and their respective phenotypic and molecular resistance patterns, the patient was treated with a combination of colistin, ceftazidime-avibactam with aztreonam, and metronidazole for 12 days. Ceftazidime-avibactam in addition with aztreonam has been shown to be efficient in vitro against metallo- $\beta$-lactamase producers despite being resistant to ceftazidime-avibactam alone. Subsequently, a regrading inflammation and a progressive wound healing of the patient was observed.

This report highlights several issues. As well admitted now, antibiotic resistance does not recognize any national border. Globalization, trade, and tourism lead altogether to a rapid and worldwide spread of resistant micro-organisms. Therefore, it is mandatory to screen for multidrugresistant bacteria and multiple of those resistant bacteria in patients being hospitalized abroad, to rapidly implement the adequate hygiene and infection control measures, and consequently prevent further nosocomial spread.

It is well known that NDM producers are endemic in India and the Middle East. The Indian subcontinent represents a huge and growing reservoir of multiple resistant bacteria. In addition, the emergence of resistance determinants to conferring resistance to all available aminoglycosides (namely the 16S rRNA methylases) has been already highlighted in Switzerland [10]. This additional resistance trait is often associated with carbapenemases of the NDM type and shall be systematically searched for, since aminoglycosides together with $\beta$-lactams and fluoroquinolones remain the main antibiotics for treating Gram-negative infections. Even though some therapeutical options may remain against such NDM-producing strain, particularly fosfomycin, nitrofurantoin, colistin [11], this report highlights the threat of pandrug resistance in Gramnegatives, even in Switzerland, is not far.

Funding This work was funded by the University of Fribourg and by the Swiss National Reference Center for Emerging Antibiotic Resistance (NARA). 


\section{Compliance with ethical standards}

Conflict of interest None to declare.

Informed consent All the authors agreed on the submitted version of the manuscript.

\section{References}

1. ecdc.europa.eu [Internet]. ECDC/EMEA Joint Technical Report. The bacterial challenge: time to react. c2019. https://ecdc.europ a.eu/sites/portal/files/media/en/publications/Publications/0909_ TER_The_Bacterial_Challenge_Time_to_React.pdf. Accessed 16 Jan 2019.

2. swissnoso.ch [Internet]. National Centre for Infections prevention; c2019. https://www.swissnoso.ch/en/what-is-it-about/healthcare -associated-infections/. Accessed 10 Jan 2019.

3. Gasser MZ, Cassini W, Kronenberg A. Attributable deaths and disability-adjusted life-years caused by infections with antibioticresistant bacteria in Switzerland. Lancet Infect Dis. 2019;19:17-8.

4. European Committee on Antimicrobial Susceptibility Testing. 2019. Breakpoint tables for interpretation of MICs and zone diameters. Version 9.0. https://www.eucast.org/fileadmin/src/media/
PDFs/EUCAST_files/Breakpoint_tables/v_9.0_Breakpoint_Table s.pdf.

5. Nordmann P, Poirel L, Dortet L. Rapid detection of carbapenemase-producing Enterobacteriaceae. Emerg Infect Dis. 2012;18:1503-7.

6. Dortet L, Poirel L, Nordmann P. Rapid detection of carbapenemase-producing Pseudomonas spp. J Clin Microbiol. 2012;50:3773-6.

7. Dortet L, Poirel L, Errera C, Nordmann P. CarbAcineto NP test for rapid detection of carbapenemase-producing Acinetobacter spp. $\mathrm{J}$ Clin Microbiol. 2014;52:2359-64.

8. Nordmann P, Jayol A, Poirel L. Rapid detection of polymyxin resistance in Enterobacteriaceae. Emerg Infect Dis. 2016;22:1038-43.

9. Nordmann P, Dortet L, Poirel L. Rapid detection of extendedspectrum $\beta$-lactamase-producing Enterobacteriaceae. J Clin Microbiol. 2012;50:3016-22.

10. Mancini S, Poirel L, Tritten ML, Lienhard R, Bassi C, Nordmann P. Emergence of an MDR Klebsiella pneumoniae ST231 producing OXA-232 and RmtF in Switzerland. J Antimicrob Chemother. 2018;73:821-3.

11. Chibabhai V, Nana T, Bosman N, Thomas T, Lowman W. Were all carbapenemases created equal? Treatment of NDM-producing extensively drug-resistant Enterobacteriaceae: a case report and literature review. Infection. 2018;46:1-13. 